\title{
Experimental Simulation of
}

\section{Retrodirective Cross-Eye Jamming}

W. P. du Plessis, J. W. Odendaal and J. Joubert

\begin{abstract}
Experimental measurements that accurately simulate the effect of a retrodirective cross-eye jammer on a monopulse radar are described. The accuracy of a recently published extended analysis of retrodirective crosseye jamming and the limitations of the conventional phase-front analysis of cross-eye jamming are illustrated by the experiments.
\end{abstract}

\section{Index Terms}

Electronic warfare, electronic countermeasures, radar countermeasures, monopulse radar, radar tracking, and radar measurements.

\section{INTRODUCTION}

Cross-eye jamming is an Electronic Attack (EA) technique that is used to induce an angular error in the radar being jammed by recreating the worst-case glint angular error [1]-[7]. Angular deception is most often required in the final stages of an engagement where a platform is attempting to protect itself against radar-guided missiles.

The monopulse indicated angle caused by a cross-eye jammer is generally assumed to be given by [1]-[5]

$$
\theta_{i} \approx \theta_{r}+\theta_{e} G_{C}
$$

where $\theta_{i}$ is the monopulse indicated angle, $\theta_{r}$ and $\theta_{e}$ are defined in Fig. 1, and the cross-eye gain is given by [3]

$$
G_{C}=\frac{1-a^{2}}{1+a^{2}+2 a \cos (\phi)}
$$

where $a$ and $\phi$ are the relative amplitude and phase shift of the two directions through a retrodirective cross-eye jammer. However, the accuracy of this result is limited because [8]:

- a number of nonlinear functions are linearised, and

- the retrodirective implementation of cross-eye jamming is ignored.

Vakin and Shustov state that (1) is only accurate when $a \leq 0.9$ or $a \geq 1.1$ and $2 \theta_{e} \leq 0.1 \theta_{B W}$ where $\theta_{B W}$ is the 3- $\mathrm{dB}$ beamwidth of the radar antenna [4], [5]. Furthermore, a number of authors state state that the error due to a cross-eye jammer is limited to $0.6 \theta_{B W}[4]-[6]$.

W. P. du Plessis is a Ph.D. student at the University of Pretoria, Pretoria, 0002, South Africa, and works for Defence, Peace, Safety and Security (DPSS), Council for Scientific and Industrial Research (CSIR), Pretoria, 0001, South Africa (e-mail: wduplessis@ieee.org).

J. W. Odendaal and J. Joubert are with the Centre for Electromagnetism at the University of Pretoria, Pretoria, 0002, South Africa. Manuscript received October 16, 2009; revised March 3, 2010. 


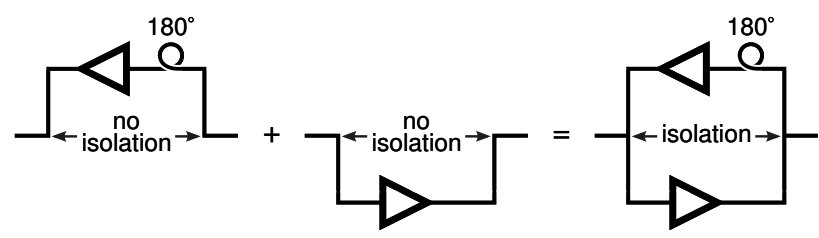

Fig. 4. The use of superposition allows a high-gain cross-eye jammer to be simulated without requiring high isolation.

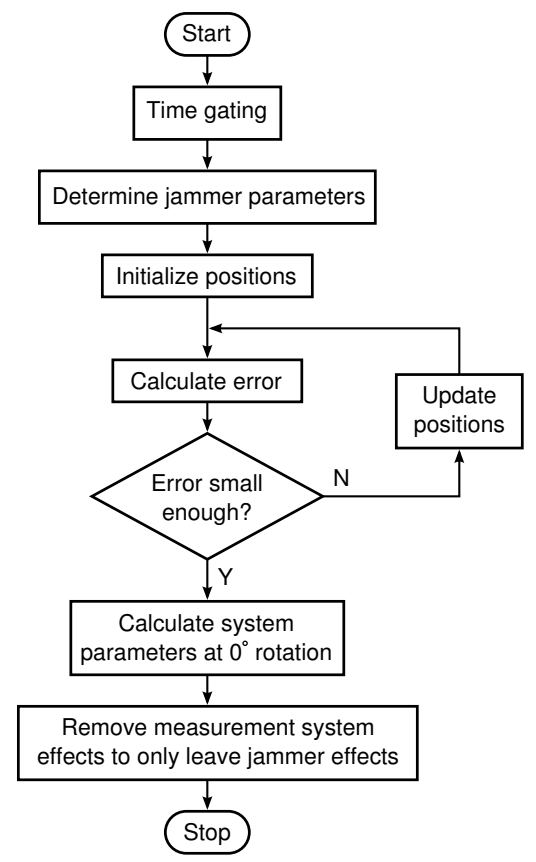

Fig. 5. A flow chart summarising the data processing procedure.

sufficient isolation between the two directions through the jammer to avoid oscillation. This problem was avoided here by combining two measurements performed using high-gain amplifiers in each direction through the jammer and then combining the two measurements to obtain the final result as shown in Fig. 4. This approach produces good results in a temperature-controlled environment when high-quality measurement equipment is used because system variations between the measurements are small.

The approximate distances between the two radar antennas and between the two jammer antennas were $70 \mathrm{~mm}$ and $550 \mathrm{~mm}$ respectively. The radar and jammer were separated by $6.3 \mathrm{~m}$, and a laser was used to align the radar and jammer antennas at zero rotation. The measurements were performed from 7.5 to $12.5 \mathrm{GHz}$.

\section{DATA PROCESSING}

The main purpose of the data processing performed on the measured backscatter data is to extract the jammer return from the measured data and is summarized by the flow chart shown in Fig. 5.

A time-domain plot of one of the raw measurements is shown in Fig. 6(a). The desired jammer return at 246 ns was extracted from the measured data using a time-gating filter to suppress the unwanted returns as shown in Fig. 6(b).

The time-gating filter was a 101-coefficient Finite Impulse Response (FIR) filter with a linear phase response. 


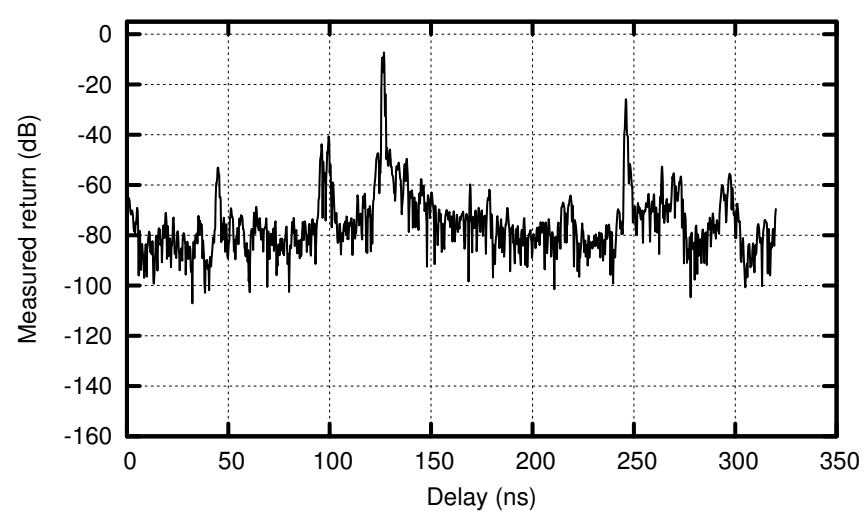

(a) Time-domain response of one of the measurements.

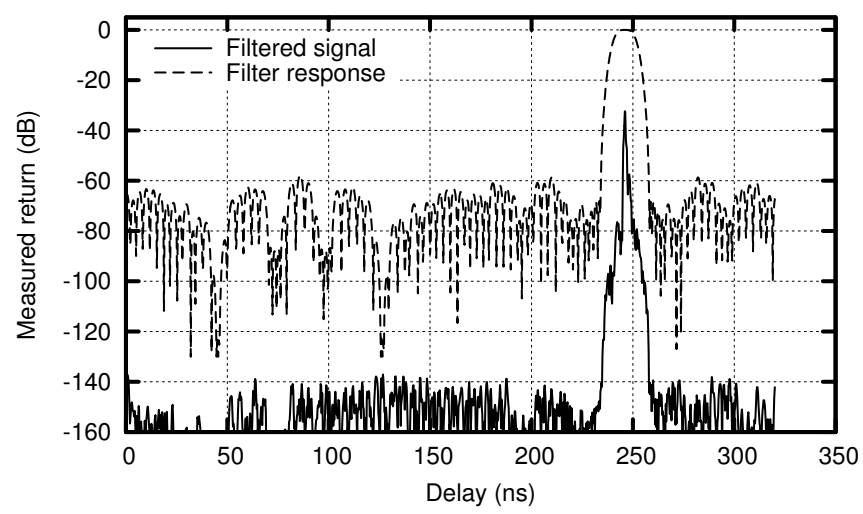

(b) Filtered time-domain response of the measurement in Fig.6(a) and timegating filter response.

Fig. 6. Typical time-domain response before and after applying time-domain filters for one of the measurements.

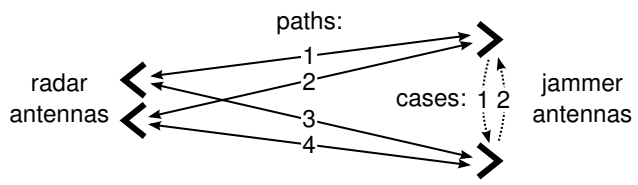

Fig. 7. The paths between the radar and jammer antennas for each of the measurements.

The zeros were positioned in pairs [10], with 98 zeros on the unit circle, and two zeros on the real axis to broaden the passband. The positions of the zeros were varied using the Nelder-Mead algorithm [11] to give the largest attenuation where the unwanted returns were strongest as shown in Fig. 6. The pairs of zeros on the unit circle and the zeros on the real axis were alternately assigned to two 51-coefficient FIR filters to minimise the effect of rounding errors.

When the top radar antenna in Fig. 7 is used for both transmission and reception, the signals travel along paths 1 and 3 in case 1, and paths 3 and 1 in case 2. Signals both transmitted and received by the bottom radar antenna travel along paths 2 and 4 in case 1, and paths 4 and 2 in case 2 . The jammer parameters can be determined directly from these measurements because the only difference between these measurements is the direction through the jammer. 
When the top radar antenna in Fig. 7 is used for transmission and the bottom radar antenna is used for reception, the signals travel along paths 1 and 4 in case 1, and paths 3 and 2 in case 2. Signals transmitted from the bottom radar antenna and received by the top radar antenna travel along paths 2 and 3 in case 1, and paths 4 and 1 in case 2 . The differences between the measurements in each case are due to the jammer parameters and the positions of the antennas. The jammer parameters can be calculated as outlined above, so the antenna positions can be determined from these measurements.

The measurement equipment effects can now be estimated as the portion of the signals that remain after the jammer and position effects are removed from the measurements. The measurement equipment effects are computed at a rotation of $0^{\circ}$ and removed from measurements at all angles to only leave the desired jammer effects.

\section{Discussion of Results}

The results are presented in Figs 8 to 10 for the case shown in Fig. 2(a) where the radar was rotated and in Fig. 11 for the case shown in Fig. 2(b) where the jammer was rotated.

The (a) and (b) portions of Figs 8 to 11 compare the measured sum- and difference-channel returns with theoretical results based on the extended analysis. These results are normalised to the return that would be received if the radar was perfectly tracking an ideal retrodirective beacon $\left(a=1, \phi=0^{\circ}\right)$. The traditional analysis only explicitly considers the angular error, and is thus not considered in the (a) and (b) portions of Figs 8 to 11. An exact monopulse processor forms its error signal from a only portion of the total differencechannel return [12], so both the total difference-channel return and the portion of the difference-channel return used for monopulse processing are shown. The measured monopulse indicated angles and the theoretical results for both the traditional and extended analyses are presented in the (c) portions of Figs 8 to 11 .

All the theoretical results were calculated assuming that the radar and jammer antenna elements were omnidirectional. The effect of this approximation is clearly seen in the sum- and difference-channel returns in Figs 8 to 11 where the measured signals drop below the theoretical signals as the radar or jammer system is rotated. As predicted by the extended analysis [9], the monopulse indicated angles in Figs 8(c) to 11(c) do not show this effect, though the noise increases at large rotations due to the antenna gain roll off. Differences between the measured data and the extended analysis are mainly due to inevitable measurement noise and to the fact that the radar antenna element patterns are not perfectly matched. Remarkably, the agreement between the measurements and the extended analysis continues to be good even well outside the sum-channel main beam (roughly $-5^{\circ}$ to $5^{\circ}$ ).

Figs 8(a) to 10(a) show that the retrodirective cross-eye jammer does not cause any angular error in the sum-channel return as predicted by the extended analysis [9]. This important result contradicts the general view that cross-eye jamming affects all radars (e.g. [1]-[3]) and means that a retrodirective cross-eye jammer will not cause an error in a radar that uses the same antenna beam for transmission and reception. For example, a conical-scan radar produces an angular error in response to an amplitude variation as its single antenna beam rotates [3]-[6]. However, the sum-channel returns in Figs 8(a) to 10(a) are symmetrical around boresight $\left(0^{\circ}\right)$, so a conical-scan radar will perfectly track the jammer. This argument obviously does not apply to Conical Scan On Receive Only (COSRO) radars which use different antenna beams for transmission and reception [1], 


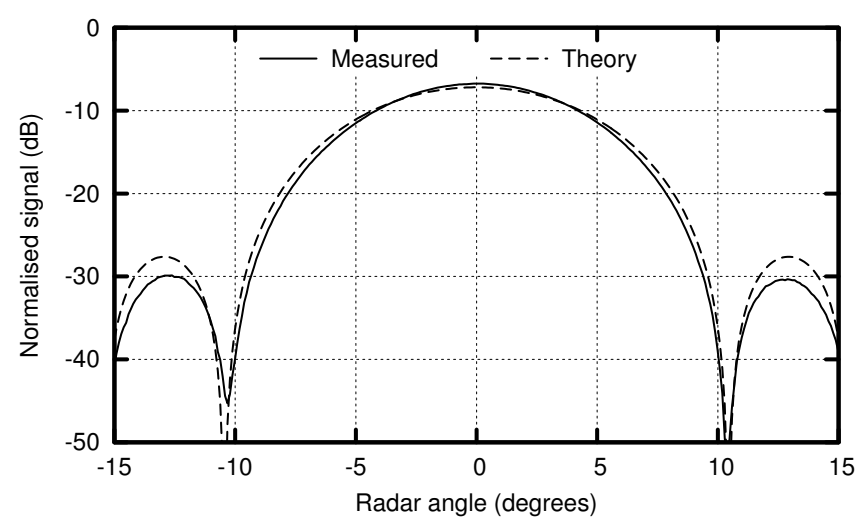

(a) Sum-channel return.

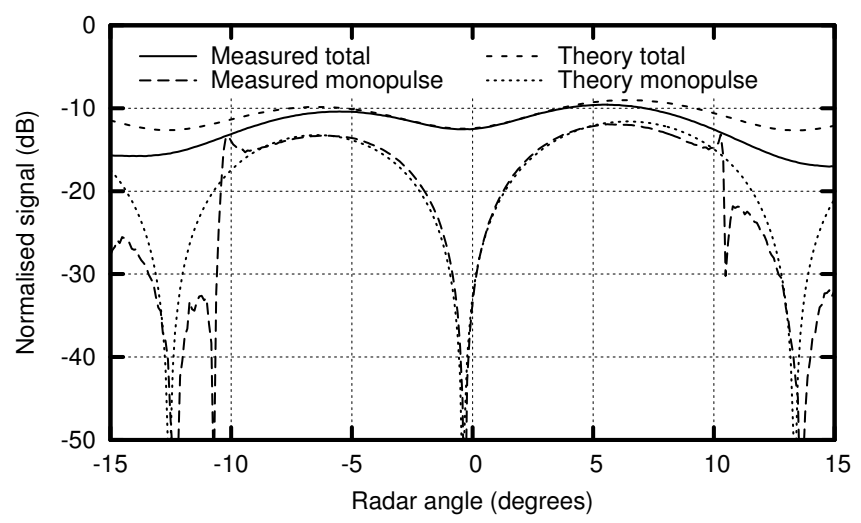

(b) The total difference-channel return and the portion of the differencechannel return used to form the monopulse indicated angle.

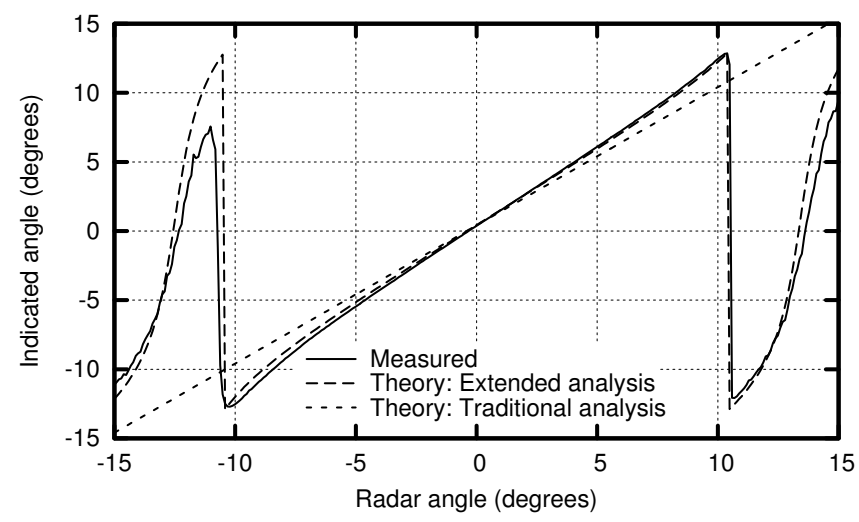

(c) Indicated angle.

Fig. 8. Monopulse signals when the radar antennas were rotated for a relative amplitude of $a=-0.72 \mathrm{~dB}$ and a relative phase shift of $\phi=120.1^{\circ}$ giving a cross-eye gain magnitude of 0.16 .

[3], [5], [6].

Despite large variations in the cross-eye gain, the total difference-channel returns in Figs 8(b) to 10(b) change very little. By contrast, the proportion of the difference-channel return used to form the monopulse error changes dramatically as predicted by the extended analysis [9].

The agreement between the measured indicated angle and the indicated angle calculated using the traditional 


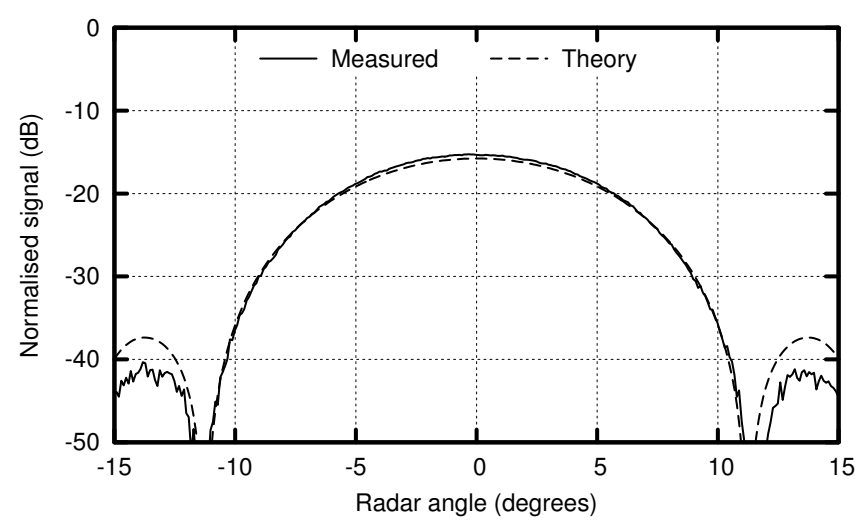

(a) Sum-channel return.

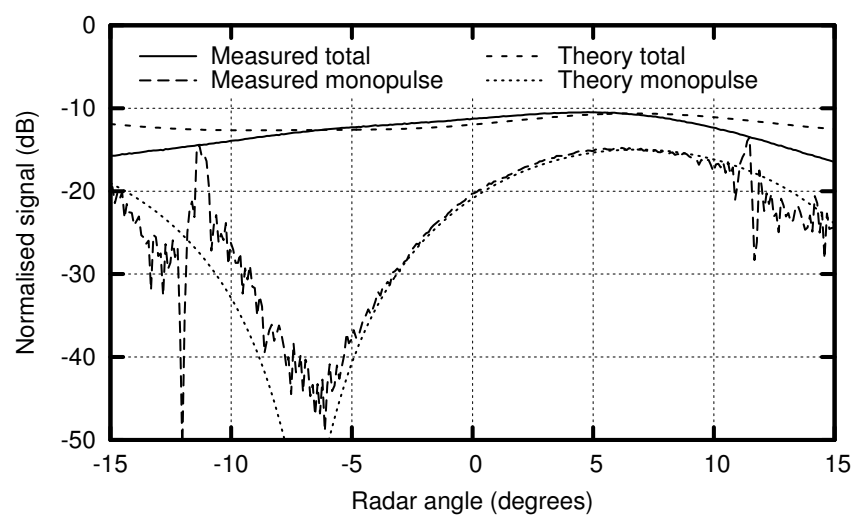

(b) The total difference-channel return and the portion of the differencechannel return used to form the monopulse indicated angle.

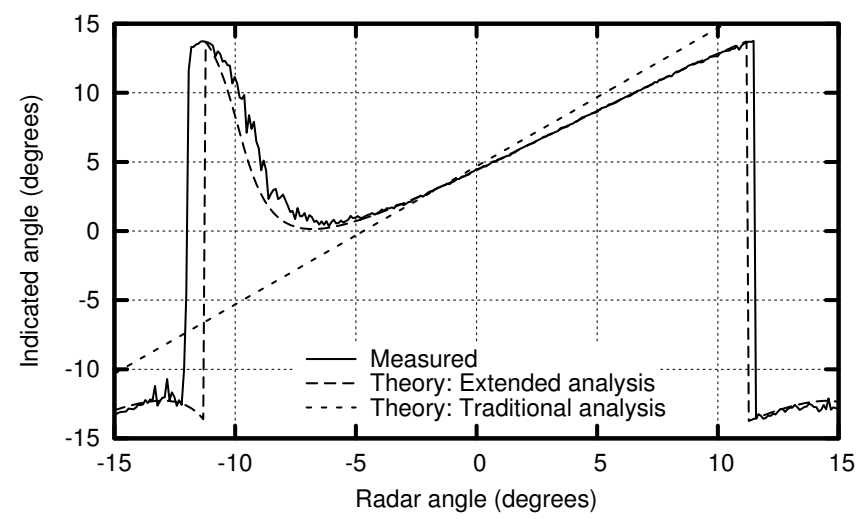

(c) Indicated angle.

Fig. 9. Monopulse signals when the radar antennas were rotated for a relative amplitude of $a=-1.17 \mathrm{~dB}$ and a relative phase shift of $\phi=200.4^{\circ}$ giving a cross-eye gain magnitude of 1.9 .

analysis decreases as the cross-eye gain magnitude increases from Fig. 8(c) to Fig. 10(c), while the extended analysis is accurate in all cases.

The traditional analysis is accurate on boresight in Figs 8 and 9 because most of the assumptions limiting the accuracy of the traditional analysis are accurate on boresight [8]. However, the traditional analysis is inaccurate even on boresight in Fig. 10 because of the large cross-eye gain magnitude. 


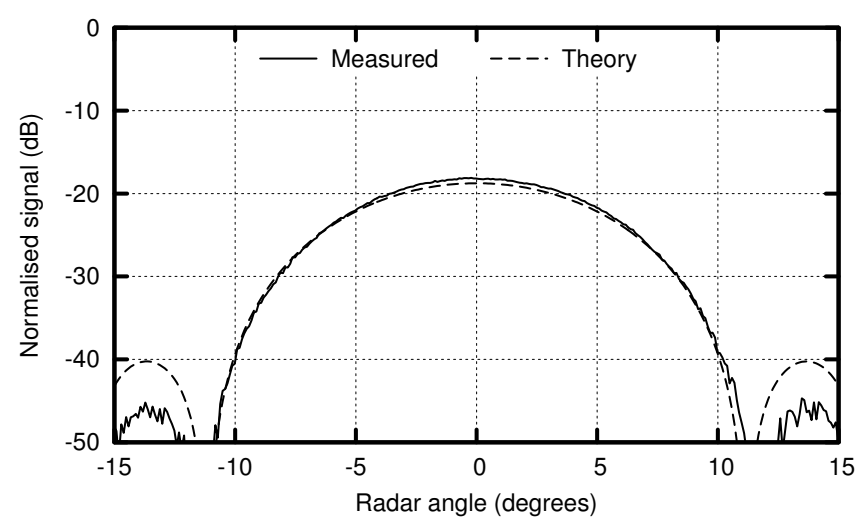

(a) Sum-channel return.

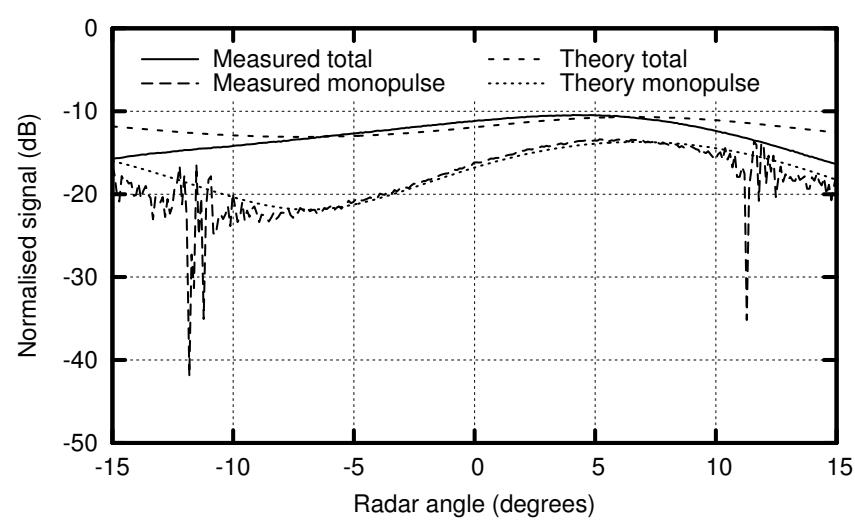

(b) The total difference-channel return and the portion of the differencechannel return used to form the monopulse indicated angle.

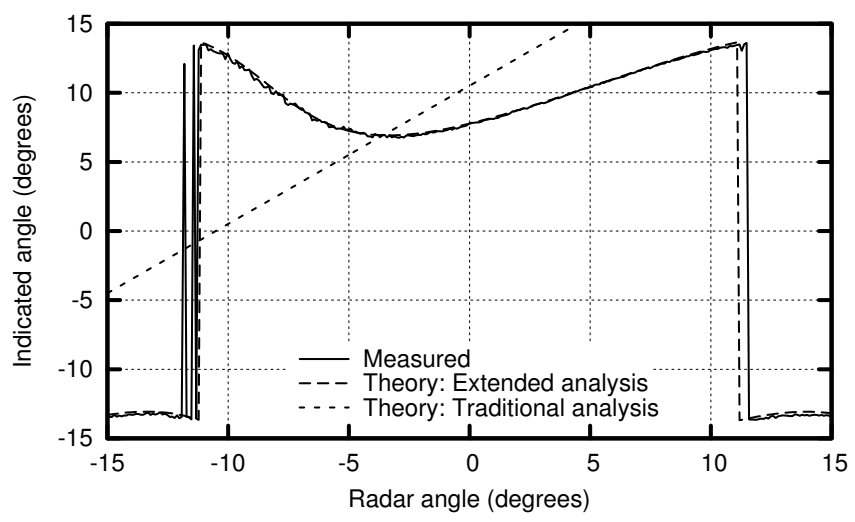

(c) Indicated angle.

Fig. 10. Monopulse signals when the radar antennas were rotated for a relative amplitude of $a=-1.34 \mathrm{~dB}$ and a relative phase shift of $\phi=192.9^{\circ}$ giving a cross-eye gain magnitude of 4.2 .

The agreement between the measurements and the traditional analysis away from boresight is accurate in Fig. 8 despite the fact that both $a=0.92$ and $2 \theta_{e}=0.45 \theta_{B W}$ violate Vakin and Shustov's bound [1], [5], showing that this bound is incomplete [13].

The accuracy of the traditional analysis away from boresight in Figs 9 and 10 is poor. The most important error is that both the measurements and the extended analysis show that the indicated angle never becomes zero 


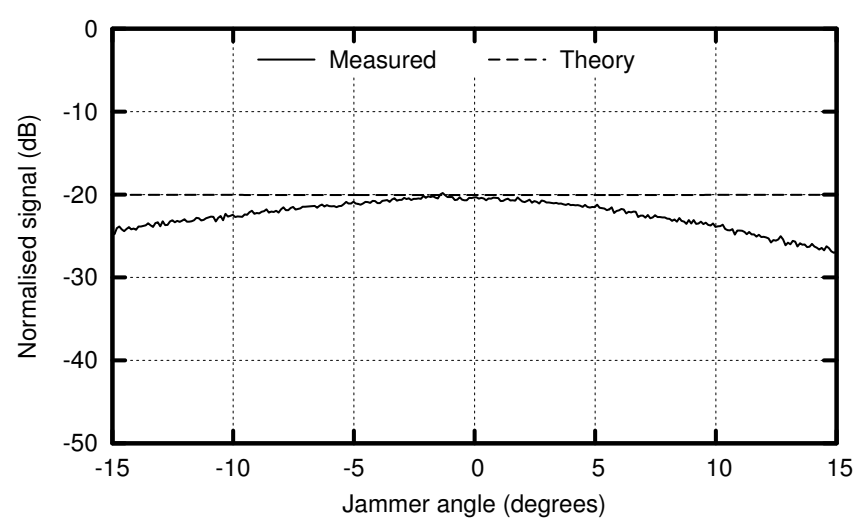

(a) Sum-channel return.

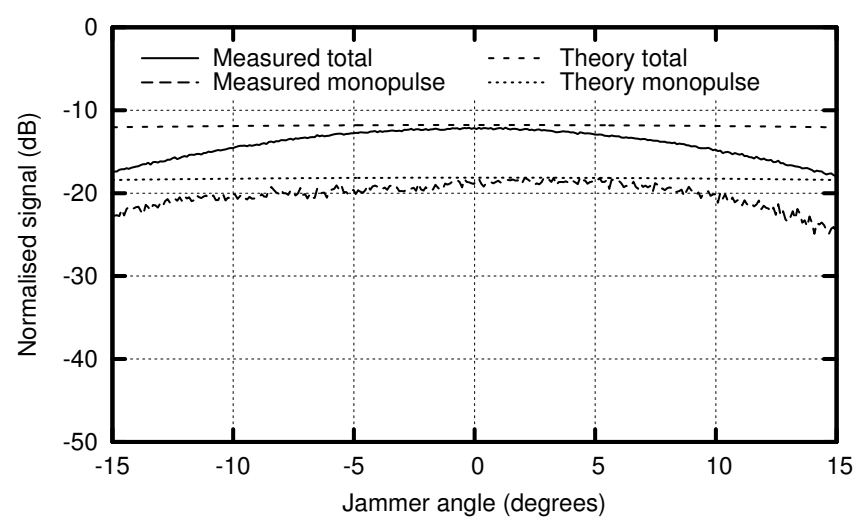

(b) The total difference-channel return and the portion of the differencechannel return used to form the monopulse indicated angle.

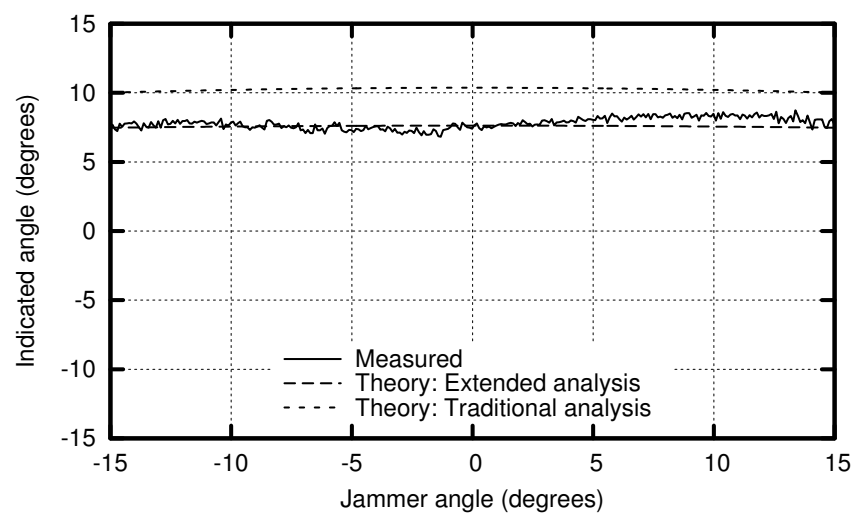

(c) Indicated angle.

Fig. 11. Monopulse signals when the jammer antennas were rotated for a relative amplitude of $a=0.84 \mathrm{~dB}$ and a relative phase shift of $\phi=190.2^{\circ}$ giving a cross-eye gain magnitude of 4.7 .

in the sum-channel main beam. This result disagrees with the widely-held view that the angular error caused by a cross-eye jammer is limited to $0.6 \theta_{B W}$ [4]-[6] and suggests that it is possible to break a monopulse radar's lock with a retrodirective cross-eye jammer that has a sufficiently large cross-eye gain magnitude.

The disagreement between the results presented here and those in the literature is due to the fact that the retrodirective implementation of cross-eye jamming is ignored in the literature. For example, the retrodirective 
nature of the jammer means that the signals for both directions through the jammer pass through both the jammer antenna elements. The total antenna gain for the two directions through the jammer is thus the same, so the only variation as the radar antenna rotates is due to the sum-channel antenna pattern which has a peak on boresight.

\section{CONClusion}

This paper presents experimental results validating the extended analysis of a retrodirective implementation of cross-eye jamming proposed in [9]. The experiments properly approximate a retrodirective cross-eye jamming scenario by using the radar system for both transmission and reception, and by simulating a retrodirective cross-eye jammer.

The measured data show excellent agreement with the extended analysis in all cases and support a number of assertions based on the extended analysis. These include the fact that a retrodirective cross-eye jammer will not produce an angular error in a radar that uses the same antenna beam for transmission and reception, and the fact that it is theoretically possible to break a monopulse radar's lock if the cross-eye magnitude gain is large enough.

A number of disagreements with the literature are highlighted, including the fact that the angular error is not limited to $60 \%$ of the radar antenna's beamwidth. These disagreements arise because the literature does not consider the retrodirective implementation of cross-eye jamming.

\section{ACKNOWLEDGMENT}

The authors would like to thank the Armaments Corporation of South Africa (Armscor) and the National Research Foundation (NRF) for supporting this research.

\section{REFERENCES}

[1] L. B. Van Brunt, Applied ECM. EW Engineering, Inc., 1978, vol. 1.

[2] N. M. Harwood, W. N. Dawber, V. A. Kluckers, and G. E. James, "Multiple-element crosseye," IET Radar Sonar Navigation, vol. 1, no. 1, pp. 67-73, February 2007.

[3] F. Neri, Introduction to electronic defense systems. Artech House, 1991.

[4] S. A. Vakin and L. N. Shustov, "Principles of jamming and electronic reconnaissance - volume I," U.S. Air Force, Tech. Rep. FTD-MT-24-115-69, AD692642, 1969.

[5] A. Golden, Radar Electronic Warfare. AIAA Inc., 1987.

[6] R. N. Lothes, M. B. Szymanski, and R. G. Wiley, Radar vulnerability to jamming. Artech House, 1990.

[7] L. Falk, "Cross-eye jamming of monopulse radar," in IEEE Waveform Diversity \& Design Conf., 4-8 June 2007, pp. $209-213$.

[8] W. P. du Plessis, "A comprehensive investigation of retrodirective cross-eye jamming," Ph.D. dissertation, University of Pretoria, 2010.

[9] W. P. du Plessis, J. W. Odendaal, and J. Joubert, "Extended analysis of retrodirective cross-eye jamming," IEEE Transactions on Antennas and Propagation, vol. 57, no. 9, pp. 2803-2806, September 2009.

[10] M. H. Hayes, Schaum's outline of theory and problems of digital signal processing, ser. Schaum's outline series. McGraw-Hill, 1999.

[11] J. A. Nelder and R. Mead, "A simplex method for function minimization," Computer Journal, vol. 7, no. 4, pp. 308-313, January 1965.

[12] S. M. Sherman, Monopulse principles and techniques. Artech House, 1984.

[13] W. P. du Plessis, J. W. Odendaal, and J. Joubert, “Tolerance analysis of cross-eye jamming systems," IEEE Transactions on Aerospace and Electronic Systems, vol. 47, no. 1, pp. 740-745, January 2011. 